$U$ such that $T[U]=\left(\begin{array}{cc}T_{1}^{(i)} & 0 \\ 0 & 0\end{array}\right)$. Then the number of (rational) integral representations of $T$ by $S$ is the same as of $T_{1}$ by $S$ and so the corresponding formula in [8] (Theorem 5) was easier to prove. For $k$, we can not always reduce $T$ to this form by a unimodular matrix over $k$, since the class number of $k$ is greater than 1 , in general.

\title{
References
}

[1] H. Braun, Zur Theorie der hermitischen Formen, Abh. Math. Sem. Hansischen Univ. 14 (1941), pp. 61-150.

[2, I, II, III] - Hermitian modular functions, I, II, III, Ann. of Math. 50 (1949), pp. 827-855, ibid. 51 (1950), pp. 92-104, ibid. 53 (1951), pp. 143-160.

[3] - Der Basissatz für hermitische Modulformen, Abh. Math. Sem. Univ. Hamburg 19 (1955), pp. 134-148.

[4] - Darstellung hermitischer Modulformen durch Poincarésche Reihen, Abh. Math. Sem. Univ. Hamburg 22 (1958), pp. 9-37.

[5] G. H. Hardy and S. Ramanujan, Asymptotic formulae in combinatory analysis. Proc. London Math. Soc. (Ser. 2) 17 (1918), pp. 75-115.

[6] P. Humbert, Théorie de la réduction des formes quadratiques définies positives dans un corps algébrique $K$ fini, Comm. Math. Helvetici 12 (1940), pp. 263-306.

[7] V. C. Nanda, On the genera of quadratic forms over algebraio number fields, 1961 (to appear).

[8] S. Raghavan, Modular forms of degree $n$ and representation by quadratio forms, Ann. of Math. 70 (1959), pp. 446-477.

[9] K. G. Ramanathan, Zeta functions of quadratio forms, Acta Arith. 7 (1961), pp. 39-69.

[10] C. L. Siegel, Über die analytische Theorie der quadratischen Formen III, Ann. of Math. 38 (1937), pp. 212-291. 622 .

[11] - On the theory of indefinite quadratic forms, Ann. of Math. 45 (1944), pp. 577.

[12] - Lectures on quadratic forms, Tata Institute of Fundamental Research, Bombay 1957.

[13] - Einführung in der Theorie der Modulfuntetionen n-ten Grades, Math. Ann. 116 (1939), pp. 617-657.

[14] W. A. Tartakowsky, La détermination de la totalité des nombres réprésentables par une forme quadratique positive à phus de quatre variables, C. R. Acad. Sci., Paris, 186 (1928), pp. 1401-1403.

TATA INSTITUTE OF FUNDAMENTAL RESEARCH, BOMBAY

Regu par la Rédaction le 9. 4. 1962

\section{Contributions to the theory of the distribution of prime numbers in arithmetical progressions III}

by

\section{S. KNAPOWSKI (Poznań)}

1. Continuing the research of [1] and [2] I shall prove in this paper some results concerning the distribution of primes $\equiv l_{1}(\bmod k)$ in comparison with those $\equiv l_{2}(\bmod k)$. Once more I shall need the conjecture (1.1) In the rectangle $0<\sigma<1,|t| \leqslant \max \left(c_{1}, k^{7}\right), s=\sigma+i t$, all $L$-functions $\bmod k$ may vanish only at points of the line $\sigma=\frac{1}{2}(\mathbf{1})$.

Writing, as usually,

$$
\pi(x, k, l)=\sum_{\substack{p=l(\bmod k) \\ p \leqslant x}} 1, \quad p \text { primes }
$$

we shall establish the following

THEOREM. Let $k \geqslant 3,0<l_{1}, l_{2}<k, l_{1} \neq l_{2},\left(l_{1}, k\right)=\left(l_{2}, k\right)=1$ and suppose (1.1) to be satisfied. Then

$$
\int_{X}^{T} \frac{\left|\pi\left(x, k, l_{1}\right)-\pi\left(x, k, l_{2}\right)\right|}{x} d x>T^{1 / 2} \exp \left(-7 \frac{\log T}{\log \log T}\right)
$$

with

for

$$
X=T \exp \left(-(\log T)^{3 / 4}\right)
$$

$$
T \geqslant \max \left(c_{2}, e^{e^{k}}\right)\left({ }^{2}\right) .
$$

Remark. In the particular case of $l_{1}=1$ one might prove a similar inequality without assuming (1.1). However, for general $l_{1}, l_{2} \mathrm{I}$ have not been able to supply any lower bound (e.g. $T^{1 / 4}$, as it used to be in the investigation of $\psi\left(x, k, l_{1}\right)-\psi\left(x, k, l_{2}\right)$ performed in [2]) for

$$
\int_{\bar{x}}^{T} \frac{\left|\pi\left(x, k, l_{1}\right)-\pi\left(x, k, l_{2}\right)\right|}{x} d x
$$

(1) $c_{1}$ and further $c_{2}, c_{3}, \ldots$ stand for positive numerical constants throughout.

(2) Compare the similar, though weaker, Theorem 3 of [2].

Acta Arithmetica viII 
or even for

$$
\max _{1 \leqslant x \leqslant T}\left|\pi\left(x, k, l_{1}\right)-\pi\left(x, k, l_{2}\right)\right|
$$

when conjecturing nothing concerning $L$-zeros.

2. Proof of this Theorem will base on the following two lemmas (for proofs see [4], p. 52, [1], p. 419 and [2], p. 327).

LEMTA 1 . Let $m$ be a non-negative number and $z_{1}, z_{2}, \ldots, z_{N}$ complex numbers such that

$$
1=\left|z_{1}\right| \geqslant\left|z_{2}\right| \geqslant \ldots \geqslant\left|z_{h}\right| \geqslant \ldots \geqslant\left|z_{N}\right|, \quad\left|z_{h}\right|>2 \frac{N}{m+N} .
$$

Then there exists an integer $\nu$ with $m \leqslant v \leqslant m+N$ such that

$$
\frac{\left|b_{1} z_{1}^{y}+b_{2} z_{2}^{\nu}+\ldots+b_{N} z_{N}^{\prime}\right|}{\left(\frac{1}{2}\left|z_{h}\right|\right)^{v}} \geqslant \min _{h \leqslant j<h_{1}}\left|b_{1}+b_{2}+\ldots+b_{j}\right|\left(\frac{1}{24 e} \cdot \frac{N}{2 N+m}\right)^{N},
$$

where $h_{1} \leqslant N$ is any integer for which $\left|z_{h_{1}}\right|<\left|z_{h}\right|-\frac{N}{m+N}$. In the case when there do not exist numbers $h_{1}$ satisfying the latter inequality, we put at the right-hand side of (2.1) $\min _{h \leqslant j \leqslant N}\left|b_{1}+b_{2}+\ldots+b_{j}\right|$ instead.

LEMTA 2. Let $k_{i} \geqslant 3,0<l_{1}, l_{2}<k, l_{1} \neq l_{2},\left(l_{1}, k\right)=\left(l_{2}, l\right)=1$. Sup pose (1.1) to be satisfied. Then there exists a number $D, \frac{1}{2} \max \left(c_{3}, k^{3}\right) \leqslant D$ $\leqslant \max \left(c_{3}, k\right)$, such that

$$
\left|\frac{1}{\varphi(k)} \sum_{(x)}\left(\bar{\chi}\left(l_{1}\right)-\bar{\chi}\left(l_{2}\right)\right) \sum_{\varrho(x)} D^{e}\left(\frac{e^{\psi e}-e^{-\psi \varphi}}{2 \psi \varrho}\right)^{2}\right| \geqslant c_{4} D \log D,
$$

where $\psi=1 / 3 D, \chi$ runs through all characters $\bmod k$ and $\varrho(\chi)$ through the zeros of $L(s, \chi)$ lying in the strip $0<\sigma<1$.

3. Pro of of the Theorem. Similarly to [1] and [2] we shall examine only the case of $k$ sufficiently large. Therefore our conjecture (1.1) can be reduced to

$$
\text { (3.1) - } \quad \prod_{x \bmod k} L(s, \chi) \neq 0 \quad \text { in } \quad \sigma>\frac{1}{2}, \quad|t| \leqslant k^{7} .
$$

We introduce the parameters

$$
\begin{gathered}
T_{1}=\frac{T}{D} e^{-2 \varphi} \quad(D, \psi \text { from Lemma } 2), \quad A=0.2 \log \log T_{1}, \\
B=\left(\log T_{1}\right)^{-0.25}, \quad m=\frac{\log T_{1}}{A+B}-\log ^{8 / 8} T_{1}\left(\log \log T_{1}\right)^{2},
\end{gathered}
$$

$r$ an integer, to be defined later, with

$$
m \leqslant r \leqslant \frac{\log T_{1}}{A+B}\left(<5 \frac{\log T_{1}}{\log \log T_{1}}\right) .
$$

Let $\alpha_{1}, \alpha_{2}, \ldots, \alpha_{\lambda}$ and $\alpha_{1}^{\prime}, \alpha_{2}^{\prime}, \ldots, \alpha_{\mu}^{\prime}$ denote all incongruent solutions $\bmod k$ of the congruences

$$
x^{2} \equiv l_{1}(\bmod k), \quad x^{\alpha} \equiv l_{2}(\bmod k)
$$

respectively. Put, further,

$$
F_{l_{1} l_{2}(s)}=\frac{1}{\varphi(k)} \sum_{(x)}\left(\bar{\chi}\left(l_{2}\right)-\bar{\chi}\left(l_{1}\right)\right) \frac{L^{\prime}}{L}(s, \chi)
$$

and start with the integral

$$
\text { (3.3) } \begin{aligned}
J_{l_{2} l_{2}=\frac{1}{2 \pi i}} & \int_{(2)}\left\{D^{s}\left(\frac{e^{\psi s}-e^{-\psi s}}{2 \psi s}\right)^{2}\left(e^{A s} \frac{e^{B s}-e^{-B s}}{2 B s}\right)^{r} F_{l_{1} l_{2}}(s)-\right. \\
& -\frac{D^{s / 2}}{2}\left(\frac{e^{\psi s / 2}-e^{-\psi s / 2}}{\psi s}\right)^{2}\left(e^{A s / 2} \frac{e^{B s / 2}-e^{-B s / 2}}{B s}\right)^{r} \times \\
& \left.\times \frac{1}{\varphi(k)}\left(\sum_{j=1}^{\mu} \sum_{(\chi)} \bar{\chi}\left(\alpha_{j}^{\prime}\right) \frac{L^{\prime}}{L}(s, \chi)-\sum_{j=1}^{\lambda} \sum_{(x)} \bar{\chi}\left(\alpha_{j}\right) \frac{L^{\prime}}{L}(s, \chi)\right)\right\} d s .
\end{aligned}
$$

Using the well-known expansion of $\frac{L^{\prime}}{L}(s, \chi)$ and writing

$$
\frac{e^{z}-e^{-z}}{2 z} \stackrel{\text { def }}{=} K(z)
$$

we obtain

$$
\begin{aligned}
J_{l_{1} l_{2}}= & \sum_{n=l_{1}(\bmod k)} \frac{\Lambda(n)}{2 \pi i} \int_{(2)} \frac{D^{s} e^{A r s}}{n^{s}} K^{2}(\psi s) K^{r}(B s) d s- \\
& -\sum_{n=l_{2}(\bmod k)} \frac{\Lambda(n)}{2 \pi i} \int_{(2)} \frac{D^{s} e^{A r s}}{n^{s}} K^{2}(\psi s) K^{r}(B s) d s- \\
& -\sum_{j=1}^{\lambda} \sum_{n=\alpha_{j}(\bmod k)} \frac{\Lambda(n)}{2 \pi i} \int_{(2)} \frac{D^{s / 2} e^{A r s / 2}}{2 n^{s}} K^{2}(\psi s / 2) K^{r}(B s / 2) d s+. \\
& +\sum_{j=1}^{\mu} \sum_{n=o_{j}^{\prime}(\bmod k)} \frac{\Lambda(n)}{2 \pi i} \int_{(2)} \frac{D^{s / 2} e^{A r s / 2}}{2 n^{8}} K^{2}(\psi s / 2) K^{r}(B s / 2) d s .
\end{aligned}
$$

We note that the first two integrals in the above formula disappear if $n$ is outside of the interval

$$
\left(X_{1} \stackrel{\text { def }}{=}\right) D e^{-2 \varphi} e^{(A-B) r}<n<D e^{2 \varphi} e^{(A+B) r}\left(\stackrel{\text { def }}{=} X_{2}\right)
$$

and similarly do the remaining integrals if $n$ is outside of

$$
X_{1}^{1 / 2}<n<X_{2}^{1 / 2} \text {. }
$$




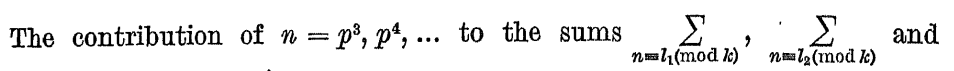
of $n=p^{2}, p^{3}, \ldots$ to $\sum_{j=1}^{\lambda} \sum_{n \equiv \alpha_{j}(\bmod k)}, \sum_{j=1}^{\mu} \sum_{n \equiv \alpha_{j}^{\prime}(\bmod k)}$, as easy to see, does not exceed $c_{5} T^{0.4}$.

Hence we have

$$
\begin{aligned}
& J_{l_{1} l_{2}}=\sum_{\substack{p=l_{1}(\bmod k) \\
X_{1} \leqslant p \leqslant X_{2}}} \frac{\log p}{2 \pi i} \int_{(2)} \frac{D^{s} e^{A r s}}{p^{s}} K^{2}(\psi s) K^{r}(B s) d s+ \\
& +\sum_{\substack{p_{2}^{2}=l_{1}(\bmod l s) \\
X_{1} \leqslant p^{2} \leqslant X_{2}}} \frac{\log p}{2 \pi i} \int_{(2)} \frac{D^{s} e^{A r s}}{p^{2 s}} K^{2}(\psi s) K^{r}(B s) d s- \\
& -\sum_{\substack{p=l_{2}(\bmod k) \\
X_{1} \leqslant p \leqslant X_{2}}} \frac{\log p}{2 \pi i} \int_{(2)} \frac{D^{s} e^{A r s}}{p^{s}} K^{2}(\psi s) K^{r}(B s) d s- \\
& -\sum_{\substack{p^{2}=l_{2}(\bmod d s) \\
X_{1} \leqslant p^{2} \leqslant X_{2}}} \frac{\log p}{2 \pi i} \int_{(2)} \frac{D^{s} e^{A r s}}{p^{2 s}} K^{2}(\psi s) K^{r}(B s) d s- \\
& -\sum_{j=1}^{\lambda} \sum_{\substack{p=a,(\bmod k) \\
X_{1}^{1 / 2} \leqslant p \leqslant X_{2}^{1 / 2}}} \frac{\log p}{2 \pi i} \int_{(2)} \frac{D^{s / 2} e^{A r s / 2}}{2 p^{8}} K^{2}(\psi s / 2) K^{r}(B s / 2) d s+ \\
& +\sum_{j=1}^{\mu} \sum_{\substack{p=a_{j}^{\prime}(\bmod k) \\
X_{1}^{1 / 2} \leqslant p \leqslant X_{2}^{1 / 2}}} \frac{\log p}{2 \pi i} \int_{(2)} \frac{D^{s / 2} e^{A r s / 2}}{2 p^{s}} K^{2}(\psi s / 2) K^{r}(B s / 2) d s+O\left(T^{0.4}\right) .
\end{aligned}
$$

We can obviously move the line of integration of the above integrals to $\sigma=0$ and substitute $s=2 w$ in the last two expressions. This makes the integrals concerned equal to

$$
\int_{(o)} \frac{D^{w} e^{A r w}}{p^{2 w}} K^{2}(\psi w) K^{r}(B w) d w
$$

i.e. equal to the ones occurring under sums $\sum_{\substack{p_{2 m}^{2 m} l_{1}(\bmod k) \\ X_{1} \leqslant p^{2} \leqslant X_{2}}}$ and $\sum_{\substack{p^{2} \operatorname{man} l_{2}(\bmod k) \\ X_{1}<p^{2}<X_{2}}}$. Since requirements $p^{2} \equiv l_{1}(\bmod k), X_{1} \leqslant p^{2} \leqslant X_{2}$ and $p \equiv \alpha_{j}(\bmod k), X_{1}^{1 / 2} \leqslant p$ $\leqslant X_{2}^{1 / 2}$ (and similarly those involved with $l_{2}$ and $a_{j}^{\prime}$ ) are clearly equivalent, we obtain finally

$$
\text { (3.4) } \begin{aligned}
J_{l_{1} l_{2}}= & \sum_{\substack{p=l_{1}(\bmod k) \\
X_{1} \leqslant p \leqslant X_{2}}} \frac{\log p}{2 \pi i} \int_{(o)} \frac{D^{s} e^{A r s}}{p^{s}} K^{2}(\psi s) K^{r}(B s) d s-\sum_{\substack{p \operatorname{ma} \\
X_{1} \leqslant p \leqslant l_{2}(\bmod k)}} \frac{\log p}{2 \pi i} \times \\
& \times \int_{(o)} \frac{D^{s} e^{A r s}}{p^{s}} K^{2}(\psi s) K^{r}(B s) d s+O\left(T^{0.4}\right) .
\end{aligned}
$$

Using Stieltjes integral we get

$$
\begin{aligned}
J_{l_{1} l_{2}}+ & O\left(T^{0.4}\right) \\
= & \int_{X_{1}}^{X_{2}}\left\{\frac{\log x}{2 \pi i} \int_{(o)} \frac{D^{s} e^{\Delta r s}}{x^{8}} K^{2}(\psi s) K^{r}(B s) d s\right\} d\left(\pi\left(x, k, l_{1}\right)-\pi\left(x, k, l_{2}\right)\right) \\
= & \left\{\left(\pi\left(x, k, l_{1}\right)-\pi\left(x, k, l_{2}\right)\right) \frac{\log x}{2 \pi i} \int_{(o)} \frac{D^{s} e^{\Delta r s}}{x^{s}} K^{2}(\psi s) K^{r}(B s) d s\right\}_{X_{1}}^{X_{2}}- \\
& -\int_{X_{1}}^{X_{2}}\left(\pi\left(x, k, l_{1}\right)-\pi\left(x, k, l_{2}\right)\right) d\left\{\frac{\log x}{2 \pi i} \int_{(o)} \frac{D^{s} e^{\Delta r s}}{x^{s}} K^{2}(\psi s) K^{r}(B s) d s\right\} \\
= & \int_{X_{1}}^{X_{2}}\left(\pi\left(x, k, l_{1}\right)-\pi\left(x, k, l_{2}\right)\right) \times \\
& \times\left\{-\frac{1}{\pi x} \int_{0}^{\infty} \cos (t(\log D+A r-\log x))\left(\frac{\sin \psi t}{\psi t}\right)^{2}\left(\frac{\sin B t}{B t}\right)^{r} d t+\right. \\
& \left.+\frac{\log x}{\pi} \int_{0}^{\infty} \sin (t(\log D+A r-\log x))\left(-\frac{t}{x}\right)\left(\frac{\sin \psi t}{\psi t}\right)^{2}\left(\frac{\sin B t}{B t}\right)^{r} d t\right\} d s .
\end{aligned}
$$

Hence

$$
\begin{aligned}
&\left|J_{l_{1} l_{2}}\right| \leqslant \int_{x_{1}}^{x_{2}} \frac{\mid \pi\left(x, k, l_{1}\right)-\pi\left(x, k, l_{2}\right)}{x} \log x d x \times \\
& \times \int_{0}^{\infty} \frac{t+1}{\pi}\left(\frac{\sin \psi t}{\psi t}\right)^{2}\left|\frac{\sin B t}{B t}\right|^{r} d t+c_{6} T^{0.4} .
\end{aligned}
$$

Noting that

$$
\begin{aligned}
\int_{0}^{\infty} \frac{t+1}{\pi}\left(\frac{\sin \psi t}{\psi t}\right)^{2} \mid & \left|\frac{\sin B t}{B t}\right|^{r} d t \leqslant \frac{1}{\pi}\left(\int_{0}^{\infty} t\left|\frac{\sin B t}{B t}\right|^{r} d t+\int_{0}^{\infty}\left(\frac{\sin \psi t}{\psi t}\right)^{2} d t\right) \\
& \leqslant \frac{1}{\pi}\left(\frac{1}{B^{2}} \int_{0}^{\infty}\left|\frac{\sin u}{u}\right|^{r} u d u+\frac{1}{\psi} \int_{0}^{\infty}\left(\frac{\sin u}{u}\right)^{2} d u\right)<(\log T)^{1 / 2},
\end{aligned}
$$

further, by (3.2), that

$X_{2}=D e^{(A+B) r+2 \psi} \leqslant D e^{2 \psi} T_{1}=T$,

$X_{1}=D e^{(A-B) r-2 \psi} \geqslant D \exp \left(-2 \psi-2 B r+\log T_{1}-(A+B) \log ^{3 / 8} T_{1}\left(\log \log T_{1}\right)^{2}\right)$

$$
>T \exp \left(-4 \psi-10 \frac{\left(\log T_{1}\right)^{0.75}}{\log \log T_{1}}-\log ^{3 / 8} T_{1}\left(\log \log T_{1}\right)^{3}\right)>T \exp \left(-(\log T)^{0.75}\right),
$$


we get

$$
\left|J_{l_{1} s}\right| \leqslant(\log T)^{3 / 2} \int_{\bar{X}}^{T} \frac{\left|\pi\left(x, k, l_{1}\right)-\pi\left(x, k, l_{2}\right)\right|}{x} d x+c_{6} T^{0.4}
$$

with

$$
X=T \exp \left(-(\log T)^{0.75}\right) .
$$

4. As in [1] and [2] we consider the infinite broken line $U$, lying in

and such that

$$
\frac{1}{30} \leqslant \sigma \leqslant \frac{1}{20}
$$

$$
\left|\frac{L^{\prime}}{L}(s, \chi)\right| \leqslant c_{7} \log ^{2}(k(|t|+1)), \quad \chi \bmod k
$$

on $U$.

Applying the theorem of residues to the integral (3.3) we get

$$
\begin{aligned}
& J_{l_{1} l_{\mathrm{s}}}=\frac{1}{\varphi(k)} \sum_{(\chi)}\left(\bar{\chi}\left(l_{2}\right)-\bar{\chi}\left(l_{1}\right)\right) \sum_{\varrho=\varrho(x)>U} D^{\varrho} e^{A r_{e}} K^{2}(\psi \varrho) K^{r}(B \varrho)- \\
& -\frac{1}{2 \varphi(k)} \sum_{j=1}^{\mu} \sum_{(\chi)} \bar{\chi}\left(\alpha_{j}^{\prime}\right) \sum_{\varrho=\varrho(x)>U} D^{e / 2} e^{A r e / 2} K^{2}(\psi \varrho / 2) K^{r}(B \varrho / 2)+ \\
& +\frac{1}{2 \varphi(k)} \sum_{j=1}^{\lambda} \sum_{(x)}^{\top} \bar{\chi}\left(\alpha_{j}\right) \sum_{e=e(x)>V} D^{e / 2} e^{\Delta r_{e} / 2} K^{2}(\psi \varrho / 2) K^{r}(B \varrho / 2)+ \\
& +\frac{1}{2} D^{1 / 2} e^{\Delta r / 2} \frac{\mu-\lambda}{\varphi(k)} K^{2}(\psi / 2) K^{r}(B / 2)+O\left(T^{0.48}\right)
\end{aligned}
$$

( $\varrho>U$ means that the $\varrho$ 's are to be taken to the right of $U$ ). The contribution of the $\varrho^{\prime}$ s with $\left|\Im_{\varrho}\right|>Y \stackrel{\text { def }}{=} \log ^{8 / 8} T_{1}$ will not exceed $c_{8} T^{0.48}$, whence all infinite series in the above formula can be reduced to sums $\sum_{\substack{\left|\beta_{0}\right| \leqslant Y \\ \rho>U}}$ Let $\varrho_{1}=\frac{1}{2}+i \gamma_{1}$ be that zero from $0<\sigma<1,|t| \leqslant k^{0.5}$ which has the greatest absolute imaginary part. We have then (see [1], (4.8))

$$
|K(B \varrho)| \geqslant\left|K\left(B \varrho_{1}\right)\right|
$$

for all zeros $\varrho=\frac{1}{2}+i \gamma,|\gamma| \leqslant\left|\gamma_{1}\right|-1$. Let, further, $\varrho_{2}=\frac{1}{2}+i \gamma_{2}$ be the zero in $|t| \leqslant 2 k^{6.5}$ with maximal $\gamma_{1}^{\prime}$. Lastly, denoting by $E$ the set of $\varrho=\varrho(\chi) \bmod k,|\Im \varrho| \leqslant Y, \varrho>U$ plus number $\frac{1}{2}$, we introduce the number $\omega \in E, \omega=u_{0}+i v_{0}$ such that

$$
\max _{z \in E}\left|e^{A x} K(B z)\right|=\left|e^{A \omega} K(B \omega)\right|
$$

Now, with aim to apply lemma 1 , we define numbers $z_{j}, b_{j}$. These will be of three categories (indices are chosen so as to have $\left|z_{1}\right| \geqslant\left|z_{2}\right| \geqslant \ldots$...

$$
\begin{gathered}
z_{j}=e^{A(\varrho-\omega)} \frac{K(B \varrho)}{K(B \omega)}, \quad|\Im \varrho| \leqslant Y, \quad \varrho>U, \\
b_{j}=\frac{1}{\varphi(\bar{k})}\left(\bar{\chi}\left(l_{2}\right)-\bar{\chi}\left(l_{1}\right)\right) D^{\circ} K^{2}(\psi \varrho) ; \\
z_{j}=e^{A(\varrho / 2-\omega)} \frac{K(B \varrho / 2)}{K(B \omega)}, \quad|\Im \varrho| \leqslant Y, \quad \varrho>U, \\
b_{j}=\frac{ \pm 1}{2 \varphi(\bar{l})} \bar{\chi}(\delta) D^{\varrho / 2} K^{2}(\psi \varrho / 2),
\end{gathered}
$$$$
\text { 2. } \quad z_{j}=e^{A(e / 2-\omega)} \frac{K(B \varrho / 2)}{K(B \omega)}, \quad|\Im \varrho| \leqslant Y, \quad \varrho>U,
$$

where $\delta$ is one of $\alpha_{\nu}$ or $\alpha_{\nu}^{\prime}$ (and \pm 1 is to be taken accordingly);

$$
\text { 3. } \quad z_{j_{0}}=e^{A(1 / 2-\omega)} \frac{K(B / 2)}{K(B \omega)}, \quad b_{j_{0}}=\frac{1}{2} D^{1 / 2} \frac{\mu-\lambda}{\varphi(k)} K^{2}(\psi / 2) .
$$

With this notation we put formula (4.1) simply as follows

with

$$
J_{l_{1} l_{1}}=\left(e^{A \omega} K(B \omega)\right)^{r} \sum_{j=1}^{N} b_{j} z_{j}^{r}+O\left(T^{0.48}\right)
$$

$$
N=\left[\log 8 / 8 T_{1}\left(\log \log T_{1}\right)^{4}\right]
$$

(if $N>1+(1+\lambda+\mu) \sum_{(x)} \sum_{\left|\xi_{e}\right| \leqslant Y, e>U} 1$ we can introduce still another category of $z_{j}$ 's $: z_{j}=b_{j}=0$ for the remaining $j$ 's). Finally we define

$$
z_{h}=e^{A\left(\varrho_{1}-\omega\right)} \frac{K\left(B \varrho_{1}\right)}{K(B \omega)}
$$

and

$$
z_{h_{1}}=e^{A\left(e_{2}-\omega\right)} \frac{K\left(B \varrho_{2}\right)}{K(B \omega)} .
$$

5. Now we shall use lemma 1 and estimate $\left|J_{l_{1} l_{2}}\right|$ from below. First of all we have

$$
\begin{aligned}
& \left|z_{h}\right|-\left|z_{h_{1}}\right|=e^{A\left(1 / 2-u_{0}\right)} \frac{\left|K\left(B \varrho_{1}\right)\right|-\left|K\left(B \varrho_{2}\right)\right|}{|K(B \omega)|} \\
& \quad \geqslant e^{-A / 2} c_{0}\left\{B^{2}\left(\gamma_{2}^{2}-\gamma_{1}^{2}\right)+O\left(B^{2} z^{6.5}\right)\right\}>c_{10} k^{13} e^{-A / 2} B^{2}=c_{10}\left(\log T_{1}\right)^{-0.6} k^{13} .
\end{aligned}
$$

On the other hand

$$
\frac{2 N}{N+m}<\frac{2 N}{m}<\frac{2 \log { }^{8 / 8} T_{1}\left(\log \log T_{1}\right)^{4}}{\log T_{1} / \log \log T_{1}}=\frac{2\left(\log \log T_{1}\right)^{5}}{\left(\log T_{1}\right)^{5 / 8}}<\frac{c_{10} k^{18}}{\left(\log T_{1}\right)^{0.6}},
$$


whence

$$
\left|z_{h}\right|-\left|z_{h_{1}}\right|>\frac{N}{N+m}
$$

(and also $\left|z_{h}\right|>2 N /(N+m)$ ). Now I assert that $z_{j}$ 's of the second category are absolutely less than $\left|z_{h_{1}}\right|$. In other words

$$
e^{A \beta / 2}|K(B \varrho / 2)|<e^{A / 2}\left|K\left(B \varrho_{2}\right)\right|
$$

for $\varrho=\beta+i \gamma, \varrho>U,|\gamma| \leqslant Y$. Using the well-known inequality (see [3], p. 295)

$$
\beta<1-\frac{c_{11}}{\max \left\{\log k, \log ^{3 / 4}(|\gamma|+3)(\log \log (|\gamma|+3))^{8 / 4}\right\}},
$$

which, owing to (1.3) and $|\gamma| \leqslant Y$, can be put as

$$
\beta<1-\frac{1}{\left(\log \log T_{1}\right)^{0.8}}
$$

we obtain

$$
e^{A \beta / 2}|K(B \varrho / 2)| \leqslant c_{12} e^{A \beta / 2} \leqslant c_{12} e^{A / 2} e^{-\left(\log \log T_{1}\right)^{0.1}},
$$

while the right-hand side of $(5.2)$ is

$$
>c_{13} e^{A / 2} \text {. }
$$

This proves (5.2). Therefore, and also by (4.2), we have

$$
\begin{aligned}
& \min _{h \leq j<h_{1}}\left|b_{1}+b_{2}+\ldots+b_{j}\right| \\
& \geqslant\left|\frac{1}{\varphi(k)} \sum_{(x)}\left(\bar{\chi}\left(l_{2}\right)-\bar{\chi}\left(l_{1}\right)\right) \sum_{\left|\Im_{e}\right| \leqslant\left|y_{1}\right|-1} D^{e}\left(\frac{e^{\psi e}-e^{-\psi \rho}}{2 \psi \varrho}\right)^{2}\right|-c_{14} \sum_{n \geqslant\left|\gamma_{1}\right|-2} \frac{D}{\psi^{2}} \cdot \frac{\log k n}{n^{2}}-\left|b_{j_{0}}\right| \\
& \geqslant\left|\frac{1}{\rho(k)} \sum_{(\chi)}\left(\bar{\chi}\left(l_{2}\right)-\bar{\chi}\left(l_{1}\right)\right) \sum_{e(\chi)} D^{e}\left(\frac{e^{\psi e}-e^{-\psi e}}{2 \psi \varrho}\right)^{2}\right|-c_{15} D^{3} \sum_{n \geqslant \frac{1}{2} k^{6.5}} \frac{\log k n}{n^{2}}-c_{16} D^{1 / 2} \\
& \geqslant\left|\frac{1}{\varphi(k)} \sum_{(\alpha)}\left(\bar{\chi}\left(l_{2}\right)-\bar{\chi}\left(l_{1}\right)\right) \sum_{\varrho(\chi)} D^{\varrho}\left(\frac{e^{\psi \varrho}-e^{-\psi \rho}}{2 \psi \varrho}\right)^{2}\right|-c_{17} k^{2.5} \log k .
\end{aligned}
$$

Hence, by lemma 2, (2.2), we get

$$
\min _{h \leqslant j<h_{1}}\left|b_{1}+b_{2}+\ldots+b_{j}\right|>c_{18} k^{3} \log k .
$$

Using now lemma 1, (4.4), (4.5), (5.1) and (5.5) we have with an appropriate $r$

(5.6) $\quad\left|J_{l_{1} l_{2}}\right| \geqslant c_{18}\left(\frac{1}{24 e} \cdot \frac{N}{2 N+m}\right)^{N} e^{A r / 2}\left|\frac{1}{2} K\left(B \varrho_{1}\right)\right|^{r}+O\left(T^{0.48}\right)$.
We obtain further the inequalities

and

$$
e^{A r / 2} \geqslant T_{1}^{1 / 2} e^{-\left(\log T_{1}\right)^{0,8}}>T^{1 / 2} e^{-(\log T)^{0.9}}
$$

$$
\left|\frac{1}{2} K\left(B \varrho_{1}\right)\right|^{r}=\left|\frac{e^{B_{\varrho_{1}}}-e^{-B_{e_{1}}}}{4 B \varrho_{1}}\right|^{r}>e^{-r}>e^{-5} \frac{\log T}{\log \log T}
$$

which together with the (rough) one

$$
\left(\frac{1}{24 e} \cdot \frac{N}{2 N+m}\right)^{N}>e^{-(\log T)^{0.5}}
$$

clearly convert $(5.6)$ to

$$
\left|J_{l_{1} l_{2}}\right|>T^{1 / 2} e^{-6 \frac{\log T}{\log \log T}} .
$$

This and (3.5) prove our assertion (1.2).

\section{References}

[1] S. Knapowski, Contributions to the theory of the distribution of prime numbers in arithmetical progressions I, Acta Arithm. 6 (1961), pp. 415-434.

[2] - Contributions to the theory of the distribution of prime numbers in arithmetical progressions II, Acta Arithm. 7 (1962), pp. 325-335.

[3] K. Prachar, Primzahlverteilung, Berlin 1957.

[4] P. Turán, Eine neue Methode in der Analysis und deren Anwendungen, Budapest 1953 .

Reçu par la Rédaction le 17. 4. 1962 